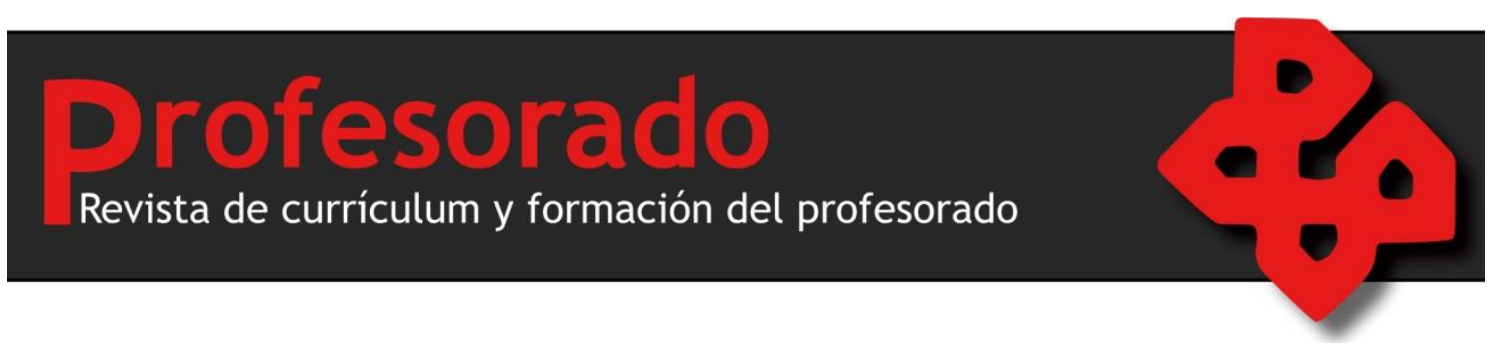

VOL.23, No4 (Octubre-Diciembre, 2019)

ISSN 1138-414X, ISSNe 1989-639X

DOI: $10.30827 /$ profesorado.v23i4.11426

Fecha de recepción: 04/11/2019

Fecha de aceptación: 11/12/2019

\title{
FORMACIÓN INICIAL DE DOCENTES COMO PRÁCTICA PARTICIPATIVA: ELABORACIÓN DE UN DICCIONARIO POLIFÓNICO
}

Initial teacher training as a participatory practice: constructing a polyphonic dictionary

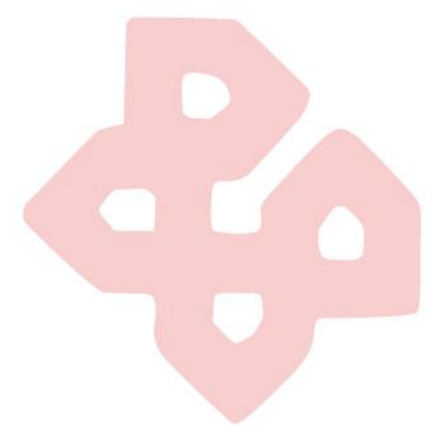

\author{
Teresa Susinos, Ángela Saiz-Linares, Julia Ruiz-López \\ y Noelia Ceballos \\ Universidad de Cantabria \\ E-mail: susinost@unican.es; saizla@unican.es; \\ ruizlj@unican.es; noelia.ceballos@unican.es \\ ORCID ID: https://orcid.org/0000-0001-6385-2196; \\ https://orcid.org/0000-0001-9226-9346; \\ https: / / orcid.org/0000-0002-5972-9504; \\ https://orcid.org/0000-0001-6962-8566
}

\begin{abstract}
Resumen:
Presentamos en este artículo un proyecto de innovación desarrollado en la Universidad de Cantabria (España) con estudiantes de los Grados de Maestro en Educación Infantil y Educación Primaria. El trabajo desarrollado con el alumnado ha consistido en elaborar mediante un proceso deliberativo y participativo un diccionario crítico de los conceptos identificados por ellos como nucleares de las asignaturas. Los estudiantes, tras un proceso de deliberación conjunta, han seleccionado varios términos que constituyen las voces de nuestro diccionario. Estos términos son definidos por diversos informantes no normativos y sus visiones se recogen en el diccionario en una versión escrita y en un objeto digital que sintetiza esta definición. Los objetivos de esta innovación tienen que ver con el desarrollo de una formación inicial docente más reflexiva e inclusiva en cuanto que promueve una mayor agencia de los estudiantes en su propio proceso formativo y que reconoce el valor de un conocimiento construido a partir de una gran diversidad de voces y perspectivas. El diccionario nace con una vocación de apertura, como una iniciativa que aspira a ser compartida, replicada y multiplicada. El artículo concluye con algunas consideraciones sobre cuáles son las cualidades de este trabajo como herramienta para una formación de docentes más inclusiva.
\end{abstract}


Palabras clave: democracia, desigualdad social, educación, formación de profesores, innovación, metodología, participación, universidad

\section{Abstract:}

This paper presents an innovation project developed at the University of Cantabria (Spain) carried out with students of the Bachelor Degrees in Infant Education and Primary Education. The project developed with the students has consisted of elaborating, through a deliberative and participatory process, a critical dictionary of the concepts identified by them as nuclear of the subjects. Students, after a process of joint deliberation, have selected several terms that constitute the voices of our dictionary. These terms are defined by various non-normative informants and their visions are collected in the dictionary in a written version and in a digital object that synthesizes this definition. The objectives of this innovation have to do with the development of a more reflective and inclusive initial teacher training because it seeks to promote a greater agency of the students and recognizes the value of a knowledge built from a great diversity of voices and perspectives. The dictionary was born with an open vocation, as an initiative that aspires to be shared, replicated and multiplied. The article concludes with some considerations on what are the main qualities of this work as a tool for a more inclusive initial teacher training.

Key words: democracy, education, innovation, methodology, participation, social inequality, teacher education, university

\section{Presentación y justificación del problema (Introducción)}

El proyecto titulado «Innovar en las prácticas de las asignaturas de Educación: Elaboración de un Diccionario Interactivo y Colaborativo como recurso en abierto ${ }^{1}$ ha sido desarrollado en la Universidad de Cantabria por un grupo de cuatro docentes en varias de las asignaturas del Grado de Maestro de Educación Infantil y Educación Primaria. En él tratamos de evidenciar y discutir con los estudiantes la naturaleza compleja y a veces conflictiva de los procesos de nominación de los fenómenos sociales $y$, en este caso, de la construcción siempre parcial de los conceptos educativos que utilizamos habitualmente. Para ello nos propusimos elaborar colaborativamente un diccionario de las asignaturas implicadas en el proyecto.

Los diccionarios son propuestas socialmente construidas que albergan una mirada determinada (y consecuentemente fragmentaria y unilateral) de la realidad que tratan de ordenar y configurar. Nombrar una realidad supone asumir un campo simbólico de significados de entre las múltiples opciones disponibles y conduce finalmente a contribuir a la naturalización de un relato que olvida y silencia otras miradas alternativas (Brailovsk, 2017; Van Manen, McClelland, y Plihal, 2007). Sin embargo, como ya se ha advertido abundantemente desde las epistemologías feministas o los estudios poscoloniales (Harding, 1993; Healey y Healey, 2018), significar y nombrar la realidad es un proceso complejo y necesariamente partidario, un mismo término puede tener variaciones semánticas, pues a través de los

\footnotetext{
1 Proyecto de Innovación financiado por el Vicerrectorado de Ordenación Académica y Profesorado de la Universidad de Cantabria en la convocatoria de 2018-19.
} 
mecanismos sociales de que se sirve la nominación, sólo una de ellas adquiere la legitimidad social. Algunos autores han puesto de manifiesto la existencia de conceptos que son utilizados bajo perspectivas diversas e incluso contradictorias según los actores a los que se atienda (Healey, Flint y Harrington, 2014). Esta situación se acentúa cuando un concepto es usado por personas en diferentes contextos geográficos, lingüísticos y culturales (Healey y Healey, 2018), o como en nuestro caso, con diferente posición social y desigual capital cultural.

Este trabajo presenta a los estudiantes la complejidad epistemológica que es inherente a los contenidos que habitualmente estudian y permite que puedan reconocer en la práctica la variedad de enfoques y perspectivas que pueden coexistir sobre un mismo fenómeno o concepto educativo.

En esta línea, este trabajo busca desarrollar una cultura educativa inclusiva en la formación inicial de los docentes en cuanto que promueve el reconocimiento del otro como sujeto con capacidad para nombrar la realidad, de aportar su propio punto de vista y de co-construir un conocimiento valioso. Los estudiantes, en el proceso de elaboración de este diccionario, se sitúan ante el reto de identificar y registrar la diversidad de voces existentes en la escuela y los diferentes intereses, necesidades, conocimientos, valores y lenguajes que coexisten (Thomson y Hall, 2015; Susinos, Ceballos y Saiz, 2018; Slee, 2011). También se ha promovido la discusión de prácticas y procesos educativos desde el marco de la educación inclusiva poniendo el acento en la lucha contra los procesos de exclusión.

Esta experiencia formativa se ha organizado como un proceso de indagación sistemática y colaborativa entre los estudiantes, los docentes y otros informantes (maestros, estudiantes, familias) sobre aquellos conceptos que el alumnado identifica como nucleares en las distintas asignaturas implicadas. Estos conceptos «críticos» de la enseñanza se convierten consiguientemente en contenidos curriculares sobre los que versará la iniciativa de formación (Cook Sather, Matthews, Ntem y Leathwick, 2018). El resultado de este proceso indagatorio consistirá en construir un diccionario polifónico (a partir de las voces de informantes diversos), utilizando diferentes lenguajes de indagación y difusión, que resume y visibiliza las experiencias y conocimientos de las personas tradicionalmente silenciadas en las epistemologías universitarias.

Mostramos en el trabajo cómo esta innovación de dos años de duración puede ser vista como una oportunidad para promover una formación de docentes más reflexiva (Beavers, Orange y Kirkwood, 2017; Bruno y Dell'Aversana, 2018; Wong, 2016) y comprometida con la inclusión educativa (tanto en sus métodos como en sus contenidos), al proponerse el abordaje de conceptos relacionados con una educación para todos y construidos desde un enfoque inclusivo: atención a la diversidad, colaboración docente, relaciones entre alumnos, etc.

Desde el punto de vista formativo, esta propuesta se sustenta en la perspectiva crítica de «students as partners» (Cook Sather, Matthews, Ntem y Leathwick, 2018) que brinda oportunidades de colaboración entre docentes y alumnado (Matthews, 
2017). La propuesta contribuye a redefinir nuestra práctica de formación de docentes desde la perspectiva de la pedagogía participativa. Esto supone cambiar la cultura universitaria desde las epistemologías disciplinares y la pedagogía de la eficiencia (Pineau, 2013), hacia experiencias de aprendizaje donde los estudiantes tienen un mayor protagonismo (Fielding, 2012).

La experiencia de innovación auspicia espacios de poder compartido y relaciones horizontales entre estudiantes y docentes donde prima el diálogo y la reflexión continuos (Cook-Sather, Felten \& Bovill, 2014; Kehler, Verwoord y Smith, 2017; Matthews, 2017). Esto coadyuva a redefinir el rol habitual del alumnado que asume un rol de mayor protagonismo y mayor agencia en las decisiones curriculares, evitando la habitual jerarquización de las relaciones y las diferencias de poder de los procesos formativos, situando la reciprocidad y la complementariedad mutuas como ejes centrales de esta formación (Hermsen, Kuiper, Roelofs, \& van Wijchen, 2017).

El objetivo final de estas experiencias es el desarrollo de contextos universitarios más inclusivos y democráticos (Ainscow, 2001; Ainscow y Miles, 2008; Apple y Beane, 2005; Fielding y Moss, 2011) que brinden oportunidades de colaboración entre docentes y alumnado (Matthews, 2017) y que, en consecuencia, sitúen a los futuros maestros como «voces autorizadas» para mejorar sus propios procesos de formación y como actores políticos del escenario educativo (Rudduck y Flutter, 2007; Susinos, 2009). Igualmente, este diccionario busca identificar y poner en valor las voces de aquellos que habitualmente no están presentes en los contextos formativos universitarios, pero cuya perspectiva debe ser tenida en cuenta para avanzar en el desarrollo de una escuela inclusiva (Susinos, Ceballos y Saiz, 2018).

En concreto, este artículo se propone describir la innovación desarrollada y analizar los resultados de esta que hacen referencia a los términos elegidos por los estudiantes para el diccionario. Pretendemos en última instancia reflejar los motivos que permiten caracterizar este trabajo como un proyecto dirigido a fomentar una formación de docentes más consecuente con un modelo inclusivo.

\section{Método}

Se trata de una investigación ya finalizada que se desplegó con los estudiantes durante el curso académico 2018-19 en dos asignaturas obligatorias (Acción Tutorial y Contextos didácticos y organizativos de la Educación Infantil); en tres asignaturas del Practicum y en varios trabajos Fin de Grado de los planes de estudio de Maestro en Educación Infantil y Primaria de la Universidad de Cantabria. En ella han participado 70 estudiantes que han trabajado en grupos de 4-5 para elaborar cada uno de los conceptos que finalmente contiene el diccionario.

La elaboración del Diccionario se planteó en todo momento como un trabajo colectivo que formaba parte de los contenidos prácticos de la asignatura. Esto significa que no ha sido un proyecto individual o periférico, sino que este trabajo se extendió 
durante un tiempo sustancial en el desarrollo de la materia porque es apreciado como vehículo de construcción del conocimiento y de estructuración del currículum de las asignaturas. De igual forma, su elaboración se desarrolló según unas fases y una cronología que los alumnos conocieron previamente, con el fin de que apreciaran que era una actividad dirigida a un fin concreto al que se pretendía llegar por medio de un proceso planificado (no como fruto de la improvisación o la serendipia) (Antúnez y Gairín, 2013; Bolívar, 2014; Hopkins, 2013; Hopkins y Reynolds, 2001; Stoll, 2013). También ha existido un compromiso con un modo de trabajo colaborativo y con un proceso de toma de decisiones respetuoso con los principios de la deliberación democrática (Della Porta, 2005; Englund, 2006; Thompson, 2008; Saiz, Rodríguez y Susinos, 2019), tal y como se explicitó claramente en la primera fase del proyecto. Finalmente, el proyecto ha pretendido establecer un puente entre los contenidos teóricos de la asignatura y las experiencias prácticas que se aprecian en las escuelas o cómo estos conceptos funcionan en la acción (Rozada, 2007; Zeichner, 2010). Por ello, en el proceso de recogida de información, los estudiantes acudieron a sus colegios de prácticas para escuchar a sus informantes y definir desde su propia perspectiva el concepto del diccionario correspondiente.

Veamos cómo se articuló todo ello en las fases previstas del proyecto (Figura 1):

1. Fase 0 o de preparación del proyecto: En esta fase se incluyeron tanto los trabajos previos realizados por el equipo docente para diseñar y poner en marcha el proyecto como específicamente la presentación a las estudiantes de la propuesta. Hubo en todo este recorrido algunos elementos imprescindibles que queremos reseñar:

- Equipo docente: diseño del proyecto, elaboración de la propuesta para la convocatoria de innovación de UC, selección de los grupos de estudiantes participantes, preparación de los documentos que se entregarán en el aula, habilitación del espacio Moodle.

- Presentación en el aula del proyecto: en un primer momento, se presentó al alumnado el proyecto y se les explicó el sentido de este y el papel nuclear que iba a tener en la asignatura como parte de su formación práctica. Asimismo, se describieron las fases y cronología y la toma de decisiones correspondiente a cada momento. Este intento por mostrar que estábamos ante un proceso planificado, pensado y complejo no fue contrapuesto con la esencia abierta, deliberativa y democrática del mismo. Durante ese momento inicial, pero también a lo largo del proyecto, se remarcaron los espacios de decisión existentes, aquellas decisiones que colaborativamente íbamos tomando.

2. Fase de deliberación: Esta fase se desarrolló alrededor de la pregunta: «¿Cuáles son los asuntos pedagógicos que te preocupan sobre esta asignatura?». A partir de las múltiples respuestas que los estudiantes ofrecieron a esta pregunta se organizó un proceso de debate deliberativo que comenzó con la 
reflexión individual de cada participante. Por ello, se configuró un período de tiempo extenso para que cada estudiante pudiera responder individualmente a la pregunta (a través de la plataforma Moodle habilitada para las asignaturas). Fue en una sesión presencial posterior donde se desplegó la deliberación colaborativa con el propósito de presentar todos los conceptos propuestos, debatir, ampliar o resignificar los conceptos propuestos de forma individual. Durante este proceso deliberativo se propició la indagación sobre la experiencia escolar de las estudiantes en torno a estos conceptos elegidos. Este debate condujo a una toma de decisiones razonada que permitió a cada grupo de estudiantes elegir un solo concepto para su trabajo posterior. Decidieron en ese momento qué concepto exploraría cada grupo; quiénes serían los informantes, las voces escuchadas; y qué estrategias de indagación desplegarían para acceder a esta definición.

3. Fase de proyecto: El alumnado se organizó en grupos (4-5 personas) y emprendió su propia indagación sobre el concepto que había seleccionado. Para planificar el proceso de recogida de información, necesitaban tomar varios acuerdos conjuntos sobre qué estrategias utilizarían, cuándo realizarían la recogida de datos, quiénes serían los informantes, etc.

Posteriormente, los estudiantes analizaron y organizaron la información obtenida con dos finalidades. La primera consistió en que cada grupo hubo de ajustar la definición de su concepto en un formato común (siguiendo los epígrafes que aparecen en la tabla 1) que era homogéneo para cada término del diccionario. La segunda tarea les proponía construir un objeto digital (que podría expresarse en diferentes lenguajes: escrito, sonoro, visual o audiovisual) que compendiaba la información obtenida de sus informantes y que facilitaba el acceso a dicho concepto por una vía no tradicional (es decir, el lenguaje escrito que utiliza el diccionario).

Todos los términos se compilaron finalmente en el diccionario que fue compartido en la web (https://dixit.unican.es).

Tabla 1

¿Cómo se organiza en el diccionario la información obtenida por cada grupo?

Concepto (ej. Rutina)

1. Voces consultadas (agentes que informan sobre este término)

2. ¿Por qué hemos elegido este concepto?

3. ¿Cómo hablan de este concepto?

(metodologías utilizadas para acceder a su visión del concepto)

4. Definición de este concepto (resumen de los datos encontrados en la indagación)

5. Autoría (estudiantes autores)

6. Objeto digital

Fuente. Elaboración propia. 
4. Fase de evaluación y difusión: Para analizar y evaluar la propuesta se utilizó una metodología de estudio de caso evaluativo (Simons, 2011), cuyo propósito último es, en el caso que nos ocupa, desentrañar el valor para la formación inclusiva de la propuesta. Para hacerlo, utilizamos instrumentos de producción de datos que permiten un tratamiento cualitativo de la información (Denzin y Lincoln, 2017): observaciones participantes de las sesiones de trabajo; análisis de documentos de los productos parciales y finales elaborados por las estudiantes; cuestionario online con preguntas abiertas y grupos de discusión con estudiantes; y entrevistas semiestructuradas con docentes (Kvale, 2011). Para realizar el análisis de los datos en nuestra investigación utilizamos un sistema de codificación temática en el que definimos las categorías de análisis y los códigos (Huber, 2003). Para definir el sistema de codificación empleamos estrategias de tipo inductivo y deductivo: partimos de un esquema inicial de variables a analizar, pero el trabajo desarrollado con los datos exigió redefinir algunas de esas categorías y códigos durante el proceso de análisis (Tójar, 2006). Más específicamente, el análisis que aquí desarrollamos corresponde a las categorías de «conceptos pedagógicos», «voces informantes» y «estrategias de acceso a los universos semánticos de los informantes», que permiten enmarcar los resultados que aquí presentamos alrededor de los ámbitos que a continuación explicamos.

La difusión del trabajo a través de la web busca abrir el diálogo más allá de los espacios habituales de la academia.

Figura 1. Fases de la propuesta.

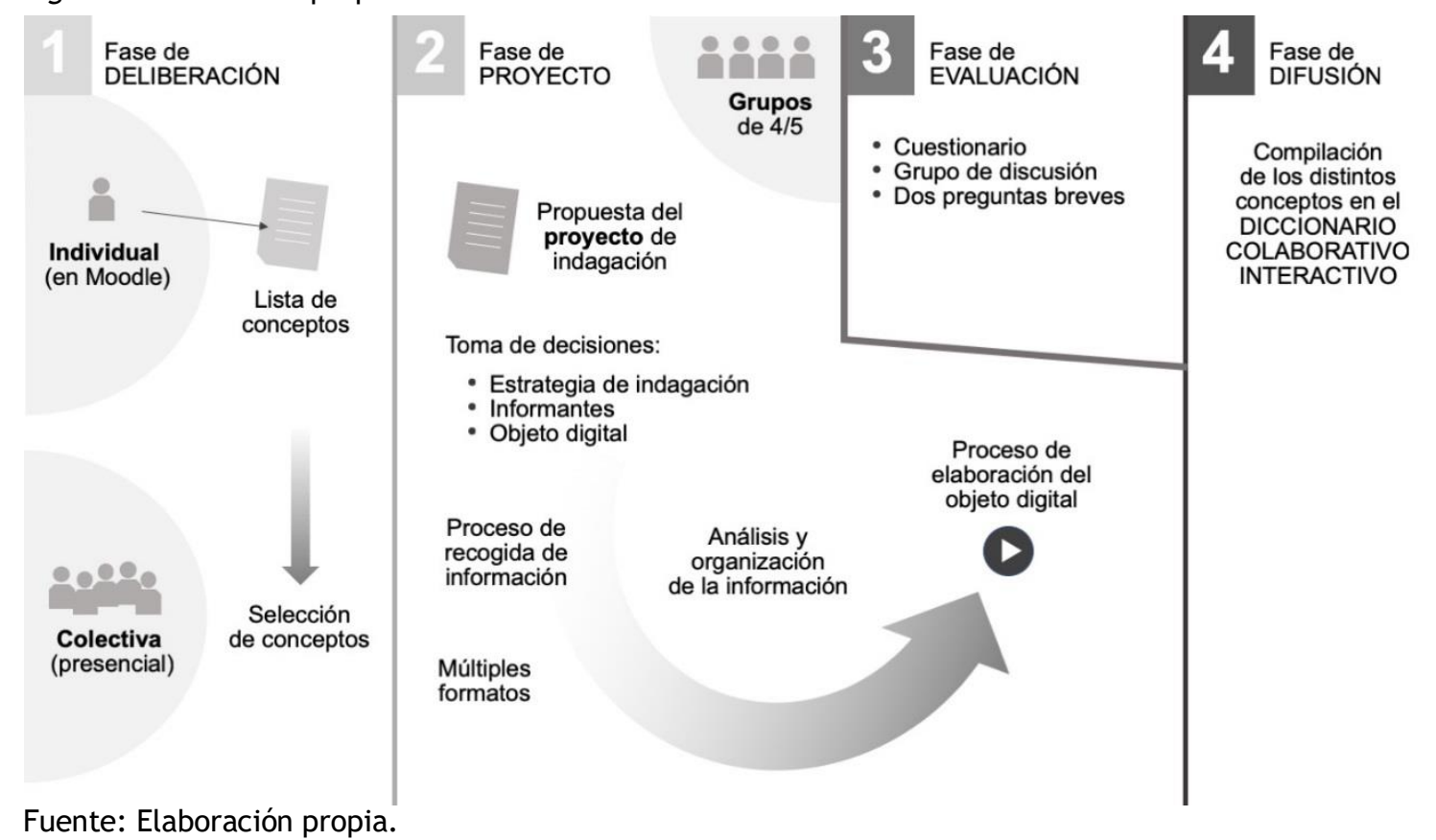




\section{Resultados}

3.1. ¿Qué conceptos eligen los estudiantes como términos nucleares en sus asignaturas?

A partir de una enorme cantidad de términos desplegados tanto en la fase individual como en la ampliación y resignificación de términos que se produce en el proceso de deliberación colectiva (234 términos en total), nuestro diccionario contiene únicamente 20 términos $(5+7+8)$. Esto nos permite imaginar la complejidad del proceso de debate y toma de decisiones que los estudiantes han tenido que llevar a cabo para finalmente elegir su propuesta. Algunos elementos que han caracterizado esta elección final pueden ser los siguientes:

- En la experiencia desarrollada en la asignatura de «Contextos didácticos y organizativos de la Educación Infantil», los términos propuestos por los estudiantes estaban desde el principio muy apegados a la práctica cotidiana de trabajo en el aula de infantil (ficha, asamblea, gomets, rincón, proyecto, juego, etc.). Otros nuevos términos surgieron en el proceso de debate colectivo (lunes y viernes, baby, colorear, alfombra, dibujar, rincón de pensar, decorar, fiestas, psicomotricidad o excursión, relaciones, espacios de aula, etc.). Sin embargo, como puede observarse, continúan siendo conceptos que se desgajan de la acción, de una visión de la Educación Infantil muy ligada a los contextos cotidianos de la vida en las aulas. Llama la atención, por ejemplo, la ausencia de conceptos ligados a cuestiones políticas, sociales e incluso epistemológicas más globales como puede ser Infancia, Educación Infantil, políticas de la Primera infancia, etc. Tampoco son propuestos otros conceptos relativos al contexto institucional del centro o que problematicen la función del docente de Educación Infantil.

- La experiencia en el «Practicum» corrobora la influencia que tiene en su elección de términos la especialidad cursada: sesión deportiva o docente especialista son conceptos elegidos por un estudiante de Educación Física; bilingüismo o interdisciplinariedad por una estudiante en la Mención de Inglés; e inclusión, dificultad de aprendizaje y autismo por otra en Pedagogía Terapéutica. Así mismo, se refleja la influencia de la práctica educativa desplegada en las escuelas en la que desarrollan su «Practicum». Por ejemplo, en una escuela en la que el dibujo y el texto libre son actividades recurrentes, estos términos aparecen en todos los estudiantes. O el caso de Yedra, la plataforma educativa de comunicación con las familias que desde hace algunos años forma parte cotidiana del quehacer de los docentes. Por otro lado, también hallamos conceptos más generales anudados con la experiencia educativa que los estudiantes de prácticas experimentaron en su historia escolar y que ahora vuelven a vivenciar en su periodo de prácticas, con una mirada desde diferente tamiz: libro de texto, maestro, reuniones, TIC...

El proceso deliberativo en el «Practicum» sirvió para seguir precisando los intereses reflexivos de los estudiantes. También permitió indagar sobre 
términos alternativos relacionados con dichos intereses que evocaran más connotaciones semánticas para los informadores y que permitieron, consecuentemente, acceder más hondamente a la constelación de significados sobre dicho interés. Por ejemplo, el término chaleco amarillo ${ }^{2}$ (se trata de una prenda que visten en los recreos y salidas algunos niños con necesidades educativas especiales), se utilizó para indagar acerca de la atención a la diversidad y la inclusión en ese centro.

- En el caso de la asignatura «Acción Tutorial» encontramos que los términos propuestos por el alumnado se orientan a conocer las funciones y roles de los agentes educativos directamente encargados de los procesos de tutorización (tutor/a, orientador/a, etc.) así como procesos educativos claramente enmarcados en el campo de estudio de la asignatura (cohesión grupal, relaciones con las familias, acogida, transición entre etapas, etc.). En esta ocasión, los conceptos propuestos no se circunscriben a la actividad del aula sino más bien a las funciones de la figura del tutor/a que exceden de esta.

En el caso de «Acción Tutorial», los conceptos que emergieron respondían a aquellos ausentes en la consulta, pero que se circunscriben al universo epistemológico de la asignatura, podemos señalar desarrollo cívico o participación del alumnado, a modo de ejemplo. En este caso, destacan dos conceptos: timbre y visión histórica de la acción tutorial. Timbre no es un concepto ligado inicialmente a la materia de la asignatura, sin embargo, es utilizado como un elemento evocador para abordar otras cuestiones de mayor calado ligado a la organización escolar como son los tiempos educativos. Por su parte, el segundo concepto, pone el énfasis en un hecho que no había surgido en el resto de materias, la necesidad de reconocer el pasado y el camino transitado en un campo de conocimiento para entender el presente.

De este apartado de resultados se puede colegir que esta variedad y divergencia de conceptos contrasta con una elección final del término elegido por cada grupo de trabajo de naturaleza bastante convencional (por ejemplo, dirección escolar, dibujo libre, cuento, espacios, docente, rutinas, etc.), que devuelve a los intereses manifestados en esa primera elección individual. Comprobamos cómo priman los asuntos vinculados con el aula y es escasa la presencia de asuntos ligados a la organización escolar. Asimismo, descuellan asuntos de carácter bastante preceptivo que dificultan una reconstrucción de las representaciones colectivas sobre la escuela

\footnotetext{
2 Chaleco amarillo. En uno de los centros educativos participantes algunos niños con necesidades educativas especiales portan un chaleco amarillo en los espacios de recreos y/o en las salidas complementarias. Esta práctica, tal y como señalan los docentes en su discurso, busca facilitar a los adultos, y también al resto de compañeros, su localización. Así mismo, indican que portar esta prenda permite visibilizar a aquellos que requieren de una «atención especial». Dentro de nuestro proyecto de innovación esta práctica ha sido ampliamente discutida con las alumnas desde los principios inclusivos cuestionando si su uso no acerca al alumnado a experimentar procesos de exclusión. Este debate suscitado en nuestro proyecto se vio nutrido de la polémica que la misma práctica ha generado en otros contextos: https://www.elmundo.es/f5/comparte/2019/04/08/5cab1b20fc6c83aa1d8b4623.html
} 
desde aproximaciones más críticas y alternativas. Tomar esta constelación de términos como indicio, nos habla de su universo semántico sobre la escuela y la docencia, de algún modo biográficamente construido durante su escolarización y reforzado durante sus estudios de magisterio. Explicitar y comprender este cosmos semántico desde la formación inicial es fundamental para afrontar un proceso formativo que contribuya a problematizar las prácticas de enseñanza y aprendizaje.

Igualmente, cabe indicar que, teniendo en cuenta la inclinación a elegir términos asaz convencionales, hemos ido introduciendo algunas mejoras para facilitar el proceso de deliberación (ej. Modificar levemente la pregunta de consulta o realizar algunos ejercicios iniciales de deliberación para acceder a los universos semánticos sobre ciertas cuestiones educativas desde términos alternativos con gran capacidad de elicitación). Igualmente, hemos instigado al estudiantado a concebir una constelación de voces más amplia que permitiera acuñar significaciones discordantes para evitar este sesgo constatado en los primeros grupos de estudiantes participantes.

Tabla 2

Conceptos que contiene el diccionario, informantes, estrategias de consulta a informantes y objetos digitales de las diferentes asignaturas.

\begin{tabular}{|c|c|c|c|c|}
\hline Asignatura & Concepto & Voces & Estrategias & $\begin{array}{c}\text { Objeto } \\
\text { digital }\end{array}$ \\
\hline \multirow{5}{*}{$\begin{array}{l}\text { Contextos } \\
\text { didácticos y } \\
\text { organizativos } \\
\text { de la } \\
\text { Educación } \\
\text { Infantil }\end{array}$} & Cuento & $\begin{array}{c}\text { Alumnado } \\
\text { de } 3,4 \text { y } 5 \text { años. }\end{array}$ & $\begin{array}{l}\text { - Representación: } \\
\text { Un país sin cuentos } \\
\text { - Terminar la historia } \\
\text { - Grupo de discusión }\end{array}$ & $\begin{array}{c}\text { Video } \\
\text { interactivo }\end{array}$ \\
\hline & Director/a & $\begin{array}{l}\text { Alumnado } \\
\text { universitario } \\
\text { del Grado de } \\
\text { Magisterio }\end{array}$ & $\begin{array}{l}\text { - Cuestionario online de } \\
\text { preguntas abiertas }\end{array}$ & Audiovisual \\
\hline & Maestra/o & $\begin{array}{c}\text { Maestras de } \\
\text { Educación Infantil }\end{array}$ & $\begin{array}{l}\text { - Entrevistas } \\
\text { - Imagen elicitadora } \\
\text { aportada por las maestras }\end{array}$ & Mapa visual \\
\hline & Recreo & $\begin{array}{c}\text { Alumnado } \\
\text { de } 5^{\circ} \text { de } \\
\text { Educación Primaria } \\
\text { y de } 4 \text { años }\end{array}$ & $\begin{array}{l}\text { Infantil: } \\
\text { - Paseo pedagógico } \\
\text { - Diálogo informal } \\
\text { Primaria: } \\
\text { - Evento consultivo }\end{array}$ & $\begin{array}{c}\text { Mapa visual } \\
\text { y grabación } \\
\text { en vídeo a } \\
\text { modo } \\
\text { explicativo. }\end{array}$ \\
\hline & Rutina & $\begin{array}{c}\text { Maestras de } \\
\text { Educación Infantil }\end{array}$ & - Entrevistas semiestructuradas & $\begin{array}{l}\text { Video- } \\
\text { animación }\end{array}$ \\
\hline \multirow[t]{2}{*}{ Practicum } & Dibujo libre & $\begin{array}{l}\text { Alumnado } \\
\text { de } 5 \text { años }\end{array}$ & $\begin{array}{l}\text { - Dibujo libre } \\
\text { - Diálogo sobre el dibujo }\end{array}$ & Mapa visual \\
\hline & Amigo/a & $\begin{array}{c}\text { Alumnado } \\
\text { de } 2^{\circ} \text { de } \\
\text { Educación Primaria }\end{array}$ & $\begin{array}{l}\text { - Pregunta de consulta individual } \\
\text { ¿Qué es un amigo para ti? } \\
\text { Recogida en un panel } \\
\text { - Buzón. ¿Qué os hacen sentir } \\
\text { vuestros amigos? } \\
\text { - Texto libre }\end{array}$ & Infografía \\
\hline
\end{tabular}


Formación inicial de docentes como práctica participativa:

\begin{tabular}{|c|c|c|c|c|}
\hline & $\begin{array}{l}\text { Profesor de } \\
\text { prácticas }\end{array}$ & $\begin{array}{l}\text { Alumnado } \\
\text { de } 3^{\circ} \text { de } \\
\text { Educación } \\
\text { Primaria }\end{array}$ & $\begin{array}{l}\text { Estrategias individuales: } \\
\text { - dibujo libre } \\
\text { - texto libre } \\
\text { Estrategias grupales } \\
\text { - grupo de discusión }\end{array}$ & Mapa visual \\
\hline & $\begin{array}{l}\text { Relaciones } \\
\text { entre iguales }\end{array}$ & $\begin{array}{l}\text { Alumnado } \\
\text { de } 5^{\circ} \text { y } 6^{\circ} \text { de } \\
\text { Educación } \\
\text { Primaria }\end{array}$ & $\begin{array}{l}\text { - Entrevistas } \\
\text { - Cuestionario escrito con } \\
\text { preguntas abiertas }\end{array}$ & Mapa visual \\
\hline & $\begin{array}{l}\text { Espacios } \\
\text { del aula }\end{array}$ & $\begin{array}{l}\text { Alumnado de } \\
1^{\circ} \text { de Educación } \\
\text { Primaria }\end{array}$ & $\begin{array}{l}\text { - Texto libre } \\
\text { - Panel de sensaciones }\end{array}$ & Infografía \\
\hline & $\begin{array}{l}\text { Chaleco } \\
\text { amarillo }\end{array}$ & $\begin{array}{l}\text { Alumnos de } \\
\text { Primaria } \\
\text { Docentes tutores } \\
\text { Especialistas } \\
\text { del apoyo }\end{array}$ & $\begin{array}{l}\text { - Dibujo libre y entrevista } \\
\text { - Collage y entrevista } \\
\text { - Mapa conceptual y entrevista }\end{array}$ & $\begin{array}{l}\text { Video- } \\
\text { animación }\end{array}$ \\
\hline \multirow[t]{8}{*}{$\begin{array}{l}\text { Acción } \\
\text { Tutorial }\end{array}$} & $\begin{array}{l}\text { Relación } \\
\text { tutor/a- } \\
\text { alumnado } \\
\text { universitario }\end{array}$ & $\begin{array}{c}\text { Alumnado } \\
\text { del } 3^{\text {er }} \text { curso } \\
\text { del Grado de } \\
\text { Magisterio en } \\
\text { Educación Infantil }\end{array}$ & $\begin{array}{l}\text { - Cuestionario online } \\
\text { - Entrevistas }\end{array}$ & Audiovisual \\
\hline & Escuela & $\begin{array}{c}\text { Intergeneracional } \\
(7,13 \\
17,27 \\
33,47, \\
59 \text { y } 90 \text { años })\end{array}$ & $\begin{array}{l}\text { - Entrevistas } \\
\text { - Foto u objeto elicitador } \\
\text { aportado por los entrevistados. }\end{array}$ & Infografía \\
\hline & Asamblea & $\begin{array}{l}\text { Alumnado } \\
\text { de } 4 \text { años }\end{array}$ & $\begin{array}{l}\text { - Asamblea de aula } \\
\text { - Dibujo }\end{array}$ & Audiovisual \\
\hline & $\begin{array}{c}\text { Período } \\
\text { de acogida }\end{array}$ & $\begin{array}{c}\text { Maestras de } \\
\text { Educación Infantil }\end{array}$ & - Entrevista & $\begin{array}{l}\text { Infografía } \\
\text { interactiva }\end{array}$ \\
\hline & Timbre & $\begin{array}{l}\text { Intergeneracional } \\
(7,12,26,43 \\
71 \text { y } 75 \text { años })\end{array}$ & - Entrevista & Infografía \\
\hline & Examen & $\begin{array}{c}\text { Alumno de } \\
6^{\circ} \text { de Educación } \\
\text { Primaria } \\
\text { y dos estudiantes } \\
\text { de } 4^{\circ} \text { ESO }\end{array}$ & - Entrevista & Infografía \\
\hline & $\begin{array}{l}\text { Relación } \\
\text { familia- } \\
\text { escuela }\end{array}$ & $\begin{array}{c}\text { Conserje } \\
\text { de un Centro de } \\
\text { Educación Infantil } \\
\text { y Primaria }\end{array}$ & - Entrevista & Audiovisual \\
\hline & $\begin{array}{l}\text { Resolución } \\
\text { de conflictos }\end{array}$ & $\begin{array}{c}\text { Intergeneracional } \\
(7,11 \\
25,26, \\
44,48 \text { años })\end{array}$ & - Entrevista & Audiovisual \\
\hline
\end{tabular}

Fuente: Elaboración propia. 


\section{2. ¿Desde qué voces se definirá el concepto (quiénes son los informantes elegidos) y con qué metodologías se accede a su visión?}

Independientemente de la asignatura en la que fijemos el análisis, encontramos que los informantes elegidos por los estudiantes en la consulta individual inicial se sitúan preponderantemente en los colectivos de alumnado, familias y docentes.

En el proceso de deliberación colectiva posterior se pudo hacer explícita la idea de que era posible añadir otras voces no normativas que inicialmente no se habían tenido en cuenta desde donde re-construir semánticamente el concepto. A pesar de este ejercicio que invitaba a incluir otros informantes menos apegados a la escuela, las propuestas de consultar a agentes periféricos o ajenos continuaron siendo escasas.

Llama la atención que en la decisión final de cada grupo dominan dos colectivos sobre los demás: el alumnado de las etapas de Educación Infantil o Primaria y los docentes de esos estudiantes. Estos han sido, sin duda, los agentes elegidos con mayor frecuencia como informantes de nuestro diccionario. Y cabe confirmar aquí que ambos colectivos, si bien tienen escasa presencia directa en la formación universitaria como voces autorizadas en la construcción del conocimiento, son sin embargo habituales informantes en los trabajos académicos. Cuando se preguntó al finalizar a los estudiantes por esta elección, ellos justifican su decisión porque cumple los criterios de accesibilidad. En nuestra opinión, necesitamos seguir ahondando en este proceso de deliberación conjunta para que puedan ser debatidas algunas elecciones convencionales como esta porque desaprovechan la oportunidad de involucrar en el escenario educativo a otros agentes, y reconstruir ciertos universos semánticos desde aproximaciones que friccionan con las miradas que tradicionalmente han tenido hegemonía en la definición de los asuntos pedagógicos.

Resulta interesante remarcar que se produce una mayor inclinación a reconocer la voz de los docentes que la del alumnado cuando la etapa es Educación Infantil. Así podemos destacar el término rutina en el cual nuestras estudiantes modifican su planteamiento inicial (indagar desde las voces de alumnado de infantil) para escuchar a las docentes. La accesibilidad no es el criterio que prevalece aquí, como sucede en otros conceptos, sino la complejidad. Consideran que dialogar con el alumnado sobre este concepto es complicado, y, aun habiendo definido con las docentes de la asignatura una estrategia de consulta ad hoc para este colectivo, optan por cambiar (presionadas, según aclaran en la evaluación del proyecto, por la calificación que obtendrán en este trabajo ya que consideran que el colectivo docente puede ofrecerles unas respuestas más acordes a un trabajo académico).

Debemos, sin embargo, señalar algunas excepciones. En la asignatura de «Acción Tutorial», encontramos que tres grupos han indagado sobre su concepto desde una mirada intergeneracional: timbre, resolución de conflictos y escuela. Para ello, se han servido de su contexto más próximo (amigos, familiares o compañeros). Por otro lado, destaca la elección del conserje como el informante que compartirá su visión sobre el concepto de relaciones familia-escuela. Esta decisión fue el resultado del diálogo entre el grupo y las docentes, quienes tenían un punto de vista diferente sobre 
si la figura del conserje era la más adecuada para indagar dicho concepto. Finalmente, el grupo de estudiantes decidió continuar con su decisión, escuchar al conserje.

Igualmente, destacaremos otro desafío que sobrevino en la asignatura de «Practicum» con el término de chaleco amarillo. Las estudiantes mostraron desde el inicio de la propuesta una predilección por consultar las voces de las familias, especialmente de aquellas cuyos hijos portaban la debatible prenda. Sin embargo, el director del centro educativo no permitió involucrar a estos agentes educativos, apelando al hecho de los conflictos que podían aparecer por abordar con los padres un tema que él consideraba delicado. En este caso, no son las estudiantes de Practicum, sino el centro educativo quien dificulta acceder a significados divergentes desde aproximaciones que pueden no armonizar con la visión que ostenta la propia escuela.

Como ya hemos descrito previamente, una vez seleccionado el concepto y los informantes de dicho término, los estudiantes tuvieron que definir el proceso de escucha. En este punto, si bien, tal y como se observa en la tabla 2, las entrevistas y grupos de discusión predominan, debemos reconocer que el alumnado ha desplegado un amplio abanico de estrategias de investigación participativa: photovoice, walking methods, eventos consultivos, dibujos, textos libres, etc. Re-construir el conocimiento sobre la enseñanza desde el saber popular o cotidiano de voces que tradicionalmente han estado excluidas del debate pedagógico requiere desplegar técnicas de investigación creativas que conecten con las experiencias cotidianas. Esta búsqueda de opciones alternativas ha supuesto un reto formativo para el alumnado que debe ser analizado en profundidad por su capacidad para movilizar otras formas de acceso y producción del conocimiento, no solo ligadas al lenguaje verbal.

\section{Discusión}

En este artículo presentamos los resultados de una experiencia de innovación docente que se inspira en la tradición de experiencias SAP (students as partners) desarrolladas en universidades internacionales (Cook-Sather, Bovill y Felten, 2014; Bergmark y Westman, 2015). Se ha desarrollado en la Universidad de Cantabria y ha tenido por objetivo la construcción colaborativa de un glosario de términos conceptualizados desde voces no convencionales y expresados también en lenguajes heterogéneos y emergentes. Estos términos son elegidos partiendo de los intereses de los estudiantes, lo cual, tal y como señalan Baron y Corbin (2012) lleva a los alumnos a sentirse inspirados por el aprendizaje. Destacamos, a continuación, las potencialidades para la formación en educación inclusiva que se derivan de esta experiencia.

En primer lugar, cabe destacar la miríada de actores implicados en esta iniciativa de formación: docentes universitarios, aspirantes a docentes, diferentes agentes educativos de la escuela, etc. Este proyecto ha partido de la asunción de que el saber pedagógico no se encuentra únicamente en los teóricos o en aquellos que tradicionalmente desarrollan investigación educativa. Por el contrario, hemos asumido 
desde el inicio que los distintos actores atesoran conocimientos y saberes educativos muy valiosos para comprender profundamente todas las aristas de la experiencia escolar. Esta reflexión está en línea con la conclusión del trabajo de Rojas, Haya y Susinos (2016), en el que presentan una experiencia formativa en magisterio cuya conclusión preponderante refiere a la necesidad de tender puentes entre los alumnos universitarios y los profesionales de la escuela. Nuestro proyecto de innovación ha sido resultado del diálogo y la deliberación colaborativa en diferentes niveles: profesores y estudiantes universitarios; estudiantes universitarios y voces «no normativas».

Este formato de construcción conjunta del conocimiento basado en el diálogo con finalidad deliberativa es una cualidad fundamental de este proyecto y contribuye a que podamos considerarlo como un instrumento dirigido a la formación inclusiva. La deliberación democrática está en el corazón de los valores y las prácticas inclusivas y tan solo puede aprenderse en toda su complejidad por medio de la práctica en contextos reales. Por tanto, consideramos que este proyecto permite a nuestros estudiantes reflexionar conjuntamente y tomar decisiones sobre su proyecto de voces para el diccionario que son a la vez coherentes con este principio inclusivo de la deliberación democrática. En otras palabras, esta propuesta se sustenta en la cocreación del currículum junto con los estudiantes, lo que en términos de Bergmark y Westman (2016) coadyuva a promover valores democráticos e inclusivos y una visión multidimensional sobre el conocimiento. Asimismo, este proceso deliberativo permite abordar el reto planteado por autores como Slee (2011) y Thomson y Hall (2015), quienes argumentan en sus estudios la necesidad de identificar y reconocer la heterogeneidad de actores que conviven dentro de la escuela y, con ello, la diversidad de intereses, necesidades, conocimientos, valores y lenguajes que coexisten.

Respecto al primer nivel dialógico, entre docentes y estudiantes, el proyecto ha estado firmemente anudado con el propósito de subvertir las relaciones jerárquicas tradicionales en la formación universitaria hacia formas más colaborativas, en coherencia con la propuesta inclusiva de «students as partners» refrendada por los trabajos de Cook-Sather (2014) y Cook-Sather, Matthews, Ntem y Leathwick (2018). Para ello se han creado espacios de trabajo compartido entre docentes y estudiantes con el fin de reflexionar conjuntamente, generando nuevos modos de relación docente-discente sustentados en el aprendizaje mutuo y en el diálogo sincero. Esto se ahíla con la propuesta de Bergmark y Westman (2016), donde defienden que estas experiencias desafían los roles tradicionales de profesor y estudiante, lo que afecta las relaciones y la jerarquía en las aulas. En consecuencia, cada uno tuvo la oportunidad de contribuir de formas diversas a los aspectos curriculares y pedagógicos de las asignaturas implicadas (Cook Sather, Matthews, Ntem y Leathwick, 2018). Igualmente, este trabajo se alinea con experiencias como la desarrollada por Prat Grau y CamachoMiñano (2018) que buscan dar voz a los estudiantes para indagar en su experiencia escolar y «ayudarles a reflexionar y repensar sobre estas prácticas pedagógicas» ( $p$. 1). En tal sentido, nuestra propuesta ha tomado como punto de partida esta biografía escolar para generar espacios de diálogo acerca de los conceptos elegidos. Tal y como señalan autores como Baron (2013) reflexionar sobre la propia experiencia escolar de los futuros docentes es fundamental si queremos formar para el cambio, pues ésta 
genera creencias implícitas muy poderosas sobre la enseñanza y el aprendizaje que necesitan hacerse explícitas. Esta cuestión es especialmente relevante cuando abordamos la formación para la inclusión. Así, por ejemplo, comprobamos cómo en el caso del «chaleco amarillo", las alumnas inicialmente comenzaron la propuesta reafirmadas en prácticas pedagógicas muy tradicionales y segregadoras (tal y como las que experimentaron cuando ellas fueron alumnas), y con una desconfianza en otro tipo de prácticas más inclusivas que normalmente se describen en las aulas universitarias. Gracias a los espacios de reflexión planteados pudieron cuestionar y re-significar las experiencias pedagógicas que vivenciaron durante su estancia en la escuela de prácticas.

Por otro lado, los futuros docentes se han enfrentado al desafío de reconocer diferentes miradas y experiencias sobre un fenómeno educativo, cuya definición normalmente diverge de aquello conceptualizado desde las voces tradicionalmente consideradas legítimas. Para ello, los estudiantes han ideado diferentes estrategias desde las que escuchar y acceder a las experiencias y saberes de aquellos colectivos que normalmente han estado silenciados o excluidos en el proceso de construcción de conocimiento pedagógico (por ejemplo: familias, niños/as y maestros/as). En tal sentido, además de las técnicas clásicas de producción de datos (como la entrevista), los estudiantes han incorporado a su acervo pedagógico estrategias de indagación más creativas e innovadoras (propias de la investigación participativa). Estas estrategias, tal y como diferentes investigaciones refrendan (Montero-Sieburth, 2011; Punch, 2002; Yates, 2010), permiten conectar con el saber cotidiano de los participantes, con sus deseos y con sus afinidades expresivas (como, por ejemplo: photovoice, walking methods, eventos consultivos, elicitación a partir de dibujos, etc.).

Igualmente, el estudiantado ha utilizado recursos expresivos heterogéneos para la construcción del objeto digital (vídeo interactivo, mapa digital, infografía, etc.). Estos canales utilizados posibilitan formas de comprensión, de expresión y de interacción más heterogéneas reconociendo, consecuentemente, a una diversidad más grande de capacidades, de intereses y de ritmos de aprendizaje, un proceso explorado en la experiencia de Rojas, Haya y Susinos (2016) en la elaboración de una guía de recursos: avanzando hacia la inclusión educativa. Reconocer otros canales comunicativos diferentes al código verbal (tradicionalmente considerado como el único válido en la escuela) para acceder y representar la realidad, enlaza, de manera firme, con los objetivos de crear una escuela inclusiva.

Este reconocimiento de la diversidad de voces legítimas para construir conocimiento o complementar y hacer más complejas las definiciones que tradicionalmente se han utilizado ha coadyuvado a controvertir las «representaciones oficiales», generando cambios en el espacio físico y/o simbólico de las personas participantes en esta iniciativa. En otras palabras, todos estos nuevos conceptos y miradas han posibilitado otra aproximación diferente a la escuela y han contribuido a comprender la infinita y variada constelación de «representaciones colectivas» que se han construido en torno a la misma, constituyendo una oportunidad para problematizar 
estas representaciones y vislumbrar la cualidad de la escuela como «construcción social» y no natural.

Finalmente, es esta una iniciativa con intención de ser compartida, replicada y multiplicada. El diccionario obtenido al finalizar el primer curso de la experiencia es parcial e incompleto y aspira a seguir construyéndose porque nace con una vocación de apertura. Por ello, se ha alojado este Diccionario Interactivo y Colaborativo en una página web (https: / /dixit.unican.es), con la idea de que constituya un recurso abierto y de que se convierta en material de consulta y trabajo en cursos posteriores, en otras asignaturas y con otros grupos de estudiantes, bajo el objetivo de seguir dialogando y reflexionando juntos y construyendo conocimiento pedagógico desde otros referentes.

\section{Referencias bibliográficas}

Ainscow, M. (2001). Desarrollo de escuelas inclusivas. Madrid: Narcea.

Ainscow, M., y Miles, S. (2008). Por una educación para todos que sea inclusiva: ¿Hacia dónde vamos ahora? Perspectivas, 38(1), 17-44.

Antúnez, S. y Gairin, J. (2013). La organización escolar: práctica y fundamentos. Barcelona: Graó.

Apple, M. y Beane, J. (2005). Escuelas democráticas. Madrid: Morata.

Baron, C. (2013). Using inquiry-based instruction to encourage teachers' historical thinking at historic sites. Teaching and Teacher Education, 35, 157-169.

Baron, P. y Corbin, L. (2012). Student engagement: Rhetoric and reality. Higher Education Research \& Development, 31(6), 759-772.

Beavers, E., Orange, A. y Kirkwood, D. (2017). Fostering critical and reflective thinking in an authentic learning situation. Journal of Early Childhood Teacher Education, 38(1), 3-18.

Bergmark, U. y Westman, S. (2016). Co-creating curriculum in higher education: promoting democratic values and a multidimensional view on learning, International Journal for Academic Development, 21(1), 28-40.

Bolívar, A. (2014). La autoevaluación en la construcción de capacidades de mejora de la escuela como Comunidad de Aprendizaje Profesional. Revista Portuguesa de Investigação Educacional, 14, 9-40.

Brailovsk, D. (2017). La pedagogía y su vocabulario. Voces de la Educación, 2(1), 5262.

Bruno, A. y Dell'Aversana, G. (2018). Reflective practicum in higher education: The influence of the learning environment on the quality of learning. Assessment \& Evaluation in Higher Education, 43(3), 348-358. 
Cook-Sather, A., Bovill, C. y Felten, P. (2014). Engaging students as partners in learning and teaching: A guide for faculty. San Francisco, CA: Josey-Bass.

Cook-Sather, A., Matthews, K., Ntem, A. y Leathwick, S. (2018). What We Talk About When We Talk About Students as Partners. International Journal for Students as Partners, 2(2), 1-9.

Denzin, N. y Lincoln, I. (2017). The SAGE handbook of qualitative research (5 edition). Thousand Oaks, CA: Sage Publications.

Della Porta, D. (2005). Deliberation in movement: Why and how to study deliberative democracy and social movements. Acta politica, 40(3), 336-350.

Englund, T. (2006) Deliberative communication: A pragmatist proposal. Journal of Curriculum Studies, 38(5), 503-520.

Fielding, M. (2012). Beyond Student Voice: Patterns of Partnership and the Demands of Deep Democracy. Revista de Educación, 359, 45-65.

Fielding, M. y Moss, P. (2011). Radical Education and the Common School: a democratic alternative. London: Routledge

Harding, S. (1993). Rethinking Standpoint Epistemology: What is 'Strong Objectivity'? En L. Alcoff y E. Potter (eds.), Feminist Epistemologies (pp. 49-82). New York: Routledge.

Healey, M. y Healey, R. (2018). «It depends»: Exploring the context-dependent nature of students as partners practices and policies. International Journal for Students as Partners, 2(1), 1-10.

Healey, M., Flint, A. y Harrington, K. (2014). Students as partners in learning and teaching in higher education. York: Higher Education Academy.

Hermsen, T., Kuiper, T., Roelofs, F. y van Wijchen, J. (2017). Without Emotions, Never a Partnership! International Journal for Students as Partners, 1(2), 1-5.

Hopkins, H. (2013). Exploding the myths of school reform. School Leadership \& Management, 33(4), 304-321.

Hopkins, D. y Reynolds, D. (2001). The Past, Present and Future of School Improvement: Toward the Third Age. British Educational Research Journal, 27(4), 459-475.

Huber, G. (2003). Introducción al análisis cualitativo de datos. En A. Medina, \& S. Castillo (Eds.), Metodología para la realización de Proyectos de Investigación y Tesis Doctorales (pp. 91-130). Madrid: Universitas.

Kehler, A., Verwood, R. y Smith, H. (2017). We are the Process: Reflections on the Underestimation of Power in Students as Partners in Practice. International Journal for Students as Partners, 1(1), 1-15. 
Kvale, S. (2011). Las entrevistas en investigación cualitativa. Madrid: Morata.

Matthews, K.E. (2017). Five Propositions for Genuine Students as Partners Practice. International Journal for Students as Partners, 1(2), 1-9.Pineau, G. (2013). Enseñar como si no cayese del cielo: epistemología de la transdisciplinariedad, la posibilidad de que los estudiantes universitarios de hoy construyan conocimiento en las aulas. Madrid: Depósito digital UAM.

Montero-Sieburth, M. (2011). Materiales en educación y alfabetización audiovisual. En A. Bautista García-Vera y H. M. Velasco Maillo (coords.), Antropología audiovisual: medios e investigación en educación (pp. 189-198). Madrid: Trotta.

Prat Grau, M. y Camacho-Miñano, M.J. (2018). La voz del futuro profesorado de educación primaria sobre sus experiencias previas en educación física: de los contextos de participación a los de exclusión. Profesorado. Revista de Currículum y Formación de Profesorado, 22(3), 433-452.

Punch, S. (2002). Research with Children: The Same or Different from Research with Adults? Childhood, 9(3), 321-341.

Rojas, S. Haya, I. y Susinos, T. (2016). Growing student voice in curriculum decisions at the university, Journal of Research in Special Educational Needs, 16(s1), 563567.

Rozada, J. M. (2007). ¿Son posibles los puentes entre la teoría y la práctica por todo el mundo demandados, sin pilares intermedios? En Romero J. y Luis A. (Eds.), La formación del profesorado a la luz de una profesionalidad democrática (pp. 4753). Santander: Consejería de Educación.

Rudduck, J. y Flutter, J. (2007). Cómo mejorar tu centro escolar dando la voz al alumnado. Madrid: Morata.

Saiz, Á., Rodríguez, C. y Susinos, T. (2019). 'I think we are still very directive': Teachers' discourses on democratic student participation. British Educational Research Journal, 45(1), 83-98.

Simons, H. (2009). Case Study Research in Practice. London: SAGE.

Slee, R. (2011). The irregular school: Exclusion, schooling and inclusive education. United Kingdom: Taylor \& Francis.

Stoll, L. (2013). Systemwide Reform Under Pressure: A Global Perspective on Learning and Change. Journal of Educational Administration, 51(4), 564-570.

Susinos, T. (2009): Escuchar para compartir. Reconociendo la autoridad del alumnado en el proyecto de una escuela inclusiva. Revista de Educación, 349, 119-136. http://b.link/EscucharParaCompartir

Susinos, T., Ceballos, N. y Saiz-Linares, A. (2018). Cuando todos cuentan. Experiencias de participación de estudiantes en las escuelas. Madrid: La Muralla. 
Thompson, D. (2008). Deliberative democratic theory and empirical political science. Annual Review of Political Science, 11, 497-520. https://doi.org/10.1146/annurev.polisci.11.081306.070555

Thomson, P. y Hall, C. (2015). 'Everyone can imagine their own Gellert': the democratic artist and. 'inclusion' in primary and nursery classrooms. Education 3-13, 43(4), 420-432. https://doi.org/10.1080/03004279.2015.1020660

Van Manen, M., McClelland, J. y Plihal, J. (2007). Naming Student Experiences and Experiencing Student Naming. En D. Thiessen y A. Cook-Sather (ed.). International Handbook of Student Experience in Elementary and Secondary School, (pp. 85-98). NY: Springer.

Wong, A. (2016). Considering reflection from the student perspective in higher education. SAGE Open, 6(1), 1-9.

Yates, L. (2010). The Story They Want to Tell, and the Visual Story as Evidence: Young People, Research Authority and Research Purposes in the Education and Health Domains. Visual Studies, 25(3), 280-291.

Zeichner, K. (2010). New epistemologies in teacher education. Rethinking the connections between campus courses and practical experiences in Teacher Education at university. Journal of Teacher Education, 61, 89-99.

Cómo citar este artículo:

Susinos, T., Saiz-Linares, A., Ruiz-López, J. y Ceballos, N. (2019). Formación inicial de docentes como práctica participativa: Elaboración de un diccionario polifónico. Profesorado. Revista de Currículum y Formación de Profesorado, 23(4), 108126. DOI: $10.30827 /$ profesorado.v23i4.11426 